\title{
NUEVAS NOTICIAS SOBRE LAS PINTURAS DE VICENTE CARDUCHO PARA EL CONVENTO DE TRINITARIOS DESCALZOS DE MADRID ${ }^{1}$
}

\author{
POR \\ M. ${ }^{a}$ CRUZ DE CARLOS VARONA
}

\begin{abstract}
This article presents new information regarding Vicente Carducho's paintings for the high altar and side altars of the Madrid Monastery of the barefoot Trinitarians as well as his twelve paintings of the Life of St. John of Mata, the founder of the Trinitarian order. Carducho's sources for the latter paintings are shown to be a series of prints of scenes from the Life of St. John of Mata published in Paris in 1633, one year before the painter signed his contract with the Trinitarian Monastery of Madrid to paint his series. The article also offers news facts regarding the fortunes of Carducho's paintings after the Disestablishment of the Monasteries in 1835.
\end{abstract}

El presente artículo pretende aportar algunos datos nuevos acerca de los cuadros realizados por Vicente Carducho para este convento madrileño en el retablo mayor y colaterales de la iglesia, y la serie de 12 lienzos sobre la vida de San Juan de Mata, fundador de la Orden trinitaria.

Se ofrecen noticias sobre las vicisitudes sufridas por los cuadros tras la Desamortización, y se demuestra la inspiración de Carducho en una serie de grabados que recogen escenas de la vida de San Juan de Mata publicados en París en 1633, es decir, en el año anterior a su contrato con el convento de Madrid para la realización de la serie sobre la vida del Fundador.

\section{El convento de trinitarios descalzos de Madrid}

El convento de la Encarnación, que así se llamaba la casa de los PP. Trinitarios descalzos en Madrid aunque popularmente fuera conocido con el nombre de Convento de Jesús debido a la imagen de Jesús Nazareno que desde 1682 recibió veneración en el mismo, es una fundación del año 1606. Anteriormente, en el año 1600 había llegado a Madrid procedente de Roma el Reformador de los trinitarios, San Juan Bautista de la Concepción, quien se estableció con algunos religiosos en una casa en la calle del Barquillo, hasta el año citado de 1606 en que comienza la fundación propiamente dicha.

Los primeros años de vida de esta comunidad religiosa en Madrid estuvieron llenos de dificultades debidas a las necesidades materiales que padecieron y fundamentalmente, a la

1 El presente artículo forma parte del trabajo de investigación «El Museo Nacional de la Trinidad: Cuadros procedentes de conventos trinitarios» presentado en septiembre de 1996 en el Departamento de Historia del Arte II de la Universidad Complutense de Madrid, y dirigido por el Dr. Alfonso E. Pérez Sánchez. Agradezco la colaboración prestada por Juan Martínez Cuesta y Conchita Romero para la realización de este artículo. 
oposición de los PP. calzados gue no vieron con buenos ojos que sus hermanos de Orden, entonces recién nacida la descalcez ${ }^{2}$, se estableciesen en Madrid a la vista de la Corte, por lo que acudieron a protestar al Consejo Real. Finalmente, el Vicario general expulsó a los religiosos de esta casa ${ }^{3}$.

Todos estos acontecimientos tuvieron lugar coincidiendo con el traslado de la Corte a Valladolid por lo que no quedaba en Madrid ninguna persona influyente que pudiese interceder en favor de la nueva fundación. Cuando se produce el nuevo traslado y la Corte queda fijada definitivamente en Madrid, el Duque de Lerma tomó el Patronato de este convento ${ }^{4}$ y en seguida procuró edificar una casa digna a los frailes descalzos ${ }^{5}$ :

Después de algunos años, se empezó a edificar la Casa en forma de convento, acudiendo a esto el Duque con liberalidad: pero como las cosas de la tierra son inconstantes y están sugetas a mudança, se mudaron de manera que no pudo proseguir lo comenzado. Vióse necesitada la Religión de acabar lo que faltaba de la vivienda, y fabricó iglesia y claustro. El edificio de los quartos es conforme a lo que comúnmente usamos; en la iglesia se excedió con sentimiento de muchos religiosos zelosos. Los prelados se han escusado con los arquitectos, el arquitecto con los prelados, y a la verdad todos tienen culpa: el artífice porque lo traçó y executó, y los Superiores porque lo consintieron ${ }^{6}$.

La descripción más completa que tenemos del convento es la realizada en 1772 por Antonio Ponz. Al exterior del edificio sólo reseña la portada de acceso, con un bajorrelieve de mármol representando a la Santísima Trinidad, de buena factura.

La iglesia, de orden dórico y no muy grande, la considera una de las mejores de Madrid en cuanto a sus proporciones, aunque según dice la capilla y tribuna en su tiempo recién construidas para la imagen de Jesús Nazareno, rompen la armonía de conjunto.

El retablo mayor y colaterales, de buen estilo, los dice realizados por Juan Gómez de Mora con pinturas de Vicente Carducho. No halla nada digno de mención en los restantes altares y retablos de la iglesia, a excepción de un grupo escultórico con el «Paso a Belén» realizado por Manuel Gutiérrez, en la primera capilla al entrar a la iglesia.

A la entrada del coro, destaca el conjunto de cuadros realizados también por Carducho con escenas de la vida de los Santos Fundadores, que anteriormente estuvieron repartidos por el cuerpo de la iglesia, pero al realizarse la tribuna de Jesús Nazareno se pusieron todos juntos aquí.

Por último se detiene en los grandes lienzos del claustro, realizados por Alonso del Arco, Francisco de Solís y Francisco Camilo con escenas de la vida de la Virgen ${ }^{7}$.

Con los diversos acontecimientos que tuvieron lugar en la primera mitad del siglo XIX, el convento sufriría la suerte de las restantes comunidades religiosas en España: resultó muy

\footnotetext{
3 Diego de la Madre de Dios (O.SS.T.), Crónica de los dezcalzos de la Santísima Trinidad, redentores de cautivos, Madrid, Juan Martín de Barrio, 1652, Vol. I, pp. 70-73.

4 El Patronato de la casa ducal de Lerma pasó posteriormente a la de los duques de Segorbe y Cardona y por último, a la casa ducal de Medinaceli que lo ejerció hasta la Desamortización.

5 No fue ésta la única ocasión en que D. Francisco Gómez de Sandoval y Rojas apoyó los proyectos de San Juan Bautista de la Concepción. Sobre este particular, véase Ventura Ginarte González, El Duque de Lerma, protector de la Reforma trinitaria, Madrid, 1982.

6 Diego de la Madre de Dios, op. cit., p. 83.

7 Antonio Ponz, Viaje de España. Madrid, Aguilar, 1988, Vol. II, p. 173.
} 1599.

2 El Breve de reforma de la Orden trinitaria había sido promulgado por Clemente VIII en 20 de agosto de 
afectado durante la Guerra de la Independencia; aunque rehabilitada después, la iglesia ya nunca pudo volver a su antiguo estado ${ }^{8}$.

Tras sufrir la Desamortización, en 1844 el edificio estaba ocupado por una escuela de equitación ${ }^{9}$.

En 1861 ya había sido cedido por el Duque de Medinaceli a las monjas de Caballero de Gracia y posteriormente a las de la Magdalena ${ }^{10}$. La parte que daba a la calle de las Huertas fue cedida por el gobierno a las Hermanas de la Caridad ${ }^{11}$.

Por último, en 1890 llegaron a ocupar el edificio los Capuchinos de San Antonio del Prado y en 1922 fue derribado, iniciándose las obras para la construcción de la Basílica de Jesús de Medinaceli que hoy se levanta en el lugar donde estuvo este convento y donde recibe culto la imagen de Jesús Nazareno que el trinitario Fr. Pedro de los Ángeles trajo a Madrid desde el Norte de África.

\section{Los cuadros de Carducho}

Vicente Carducho trabajó para este convento durante los primeros años de la década de 1630. Concretamente, el 4 de mayo de 1632 contrata la realización del retablo mayor de su iglesia, que se le paga en julio de 1634. De noviembre de este último año, data otro contrato del convento con el pintor para realizar catorce cuadros: doce de ellos, como ya vimos, constituirían una serie sobre la Vida de San Juan de Mata y los dos restantes, sobre los cuales el contrato no especifica nada, irían destinados a los retablos colaterales del Altar mayor ${ }^{12}$.

\section{a) Retablo mayor y colaterales}

El contrato entre el convento y el pintor, especifica que en el retablo mayor debía figurar la Santísima Trinidad, flanqueada por sendos lienzos representando a los Santos Fundadores de la orden trinitaria, y en la parte inferior debía representarse ... una Ystoria pequeña en cada lado del tamaño que diere la misma traça, y será de la ystoria que el Padre ministro y combento dispusiere y ordenare. Estas pinturas no han sido identificadas. Según Mary Crawford, el último autor que contempla el retablo in situ es Ceán Bermúdez. Como Cruzada no las cita al hablar de la obra de Carducho, probablemente fueron trasladadas en el período comprendido entre 1800 y 1865.

Sabemos con seguridad que en el retablo mayor de esta iglesia se efectuaron modificaciones a comienzos del siglo XVIII, que afectaron al cuadro de Carducho. Dichas modifica-

8 Ramón de Mesonero Romanos, Manual de Madrid. Descripción de la Corte y de la Villa, Madrid, Ayuntamiento, 1982, p. 156 (ed. facsímil de la de Madrid, 1832; 1.a ed., Madrid, 1831).

9 Id., Manual histórico-topográfico, administrativo y artístico de la Villa de Madrid, Madrid, Ayuntamiento, 1977 , p. 193 (ed. facsímil de la de Madrid, 1844).

to Por Orden de 23 de septiembre de 1843 el convento de trinitarios descalzos había sido cedido por derecho de reversión, al Duque de Medinaceli, según afirma Francisco Simón Segura, Contribución al estudio de la Desamortización en España. La desamortización de Mendizábal en la provincia de Madrid. Madrid, Instituto de Estudios Fiscales, 1969, p. 106.

"Ramón de Mesonero Romanos, El antiguo Madrid. Madrid, Abaco, 1976, pp. 214 y 215 (ed. facsímil de la de Madrid, 1861).

12 Mary Crawford Volk, Vicencio Carducho and Seventeenth-Century Castilian Painting. Nueva York, 1974 , pp. 242 y 243 . En esta obra se reproducen los documentos de los contratos, el primero de ellos realizado el 4 de mayo de 1632 ante Juan Martínez del Portillo (Archivo Histórico de Protocolos de Madrid, prot. 5541, fol. 123) y el segundo en 23 de noviembre de 1634 ante Juan García de Albertos (A.P.H.M., prot. 5571, fols. 886 y 887). 
ciones fueron realizadas durante la preparación de las fiestas que iban a tener lugar en la iglesia con ocasión de la declaración de autenticidad de las reliquias de San Juan de Mata y el permiso para exponerlas a la pública veneración, otorgado por la Sacra Congregación de Ritos el 6 de septiembre de 1721 y ratificado por Inocencio XIII el día 16 de dicho mes y año. Las reliquias se colocaron en el altar mayor del convento de Trinitarios descalzos de Madrid y de todo ello tenemos puntual información gracias a la Crónica de los festejos que tuvieron lugar ${ }^{13}$ :

... lo primero que se ideó fue el sitio donde se avía de colocar el Sagrado Cuerpo; y se halló tan propio que se discurrió el que la Providencia divina lo avía prevenido quasi cien años antes, para Trono de tan rica Perla. Es el retablo del Altar mayor de este templo, a juizio de los mejores artífices, de la más hermosa arquitectura, guardando en todos sus miembros la orden compuesta. El primer cuerpo, que es la Custodia y pedestal, hazen asiento a un arco de diez y siete pies de altura, en donde estaba un lienço de la Santísima Trinidad, pintura de las que no sólo se tiene la recomendación por lo antigua, sino es también por lo primorosa: discurrióse quitar este lienço y romper la pared maestra, haziendo en ella un arco igual al de la fábrica del retablo, formando dentro un camarín quadrado de la misma longitud y latitud que el arco, adornándolo por aora con variedad de lunetos, molduras y recuadros, manifestando en su exterior decencia de pizarras y plomos que hasta en esto avía de ostentar la grandeza de la perla que ocultaba 14

Continúa el P. Lucas de la Purificación describiendo el arca en que se colocaron las reliquias. Conocemos la disposición del conjunto por un grabado realizado en Viena en 1733, a partir de un cuadro pintado por el trinitario descalzo Fr. Bartolomé de San Antonio. Dicho grabado serviría de portada a otro libro sobre lo relativo al culto de las reliquias del Fundador ${ }^{15}$. En él se representa el arca de las reliquias sobre un pedestal, flanqueado por las figuras de la Caridad y la Penitencia y por sendos angelitos portando cadenas y grillos, en alusión al fin principal de la orden trinitaria que era la redención de cautivos. En la parte superior, la Santísima Trinidad, probablemente el cuadro de Carducho, colocado como telón de fondo al conjunto donde se hallaba el arca. El nuevo camarín que se hizo según acabamos de ver, era exactamente igual al arco del retablo y de su misma altura (17 pies). La altura del pedestal, según el P. Lucas de la Purificación, era de siete pies y el arca con los remates medía algo más de una vara, sobresaliendo el cuadro en la parte superior, tal como se puede apreciar en el grabado (Fig. 1).

Este cuadro de la Santísima Trinidad realizado por Carducho para el retablo mayor del convento no ha sido identificado con ninguna de las obras existentes del pintor. En el citado trabajo de Mary Crawford se planteó la posibilidad de que se tratase del San José con el Niño Jesús y la paloma del Espíritu Santo en la parte superior, obra firmada por Carducho en 1632 actualmente en el Museo de Bellas Artes de Narbonne. Aunque ląs medidas expresadas en el contrato no coinciden con las de este lienzo (el cuadro para este retablo debía

${ }_{13}$ Lucas de la Purificación (O.SS.T.), Ave María Santísima. Historia del sagrado cuerpo del Glorioso San Juan de Mata, Madrid, 1723.

14 Ibid., p. 91.

15 Fr. José de Santa María (O.SS.T.), Phoenix redivivus, Viena, 1733. Al pie del grabado leemos: «F. Bartl. a S. Ant. Trinit. disc. pinx. Madriti./I. et A. Schmuzer sc. Vien».

Sobre Fr. Bartolomé de San Antonio, véase Cean Bermúdez, Diccionario, Madrid, Reales Academias de la Historia y de Bellas Artes, 1965, Vol. IV, p. 318. 
medir 17 pies de alto, y el existente en Narbonne tiene unos 7 pies de altura, aprox., además de no ser de medio punto) considera esta autora que la fecha de realización, el tema representado, y las similitudes estilísticas de esta obra con los cuadros de la serie de San Juan de Mata constituyen puntos favorables para tal identificación. Sin embargo, en una carta de pago que ella misma reproduce, fechada en 29 de julio de 1634, se dice que el pago se efectúa al pintor ... por la ocupación que el dicho Vizencio tiene en la pintura de la Santísima Trinidad que a de ser para el retablo del dicho conbento... Por tanto, si en 1634 todavía está ocupado en la pintura de la Santísima Trinidad, no puede tratarse de la del Museo de Narbonne, firmada como hemos visto en $1632^{16}$.

El retablo mayor tuvo que ser desmontado y trasladado probablemente en 1836, o en fecha inmediatamente anterior, pues en el inventario realizado en el convento en abril de este último año no se cita ninguna pintura en el altar mayor, que por entonces estaba ocupado por una talla de Jesús Nazareno, flanqueada por otras dos que representaban a San Félix de Valois y el entonces Beato Miguel de los Santos ${ }^{17}$.

En cuanto a los cuadros de Carducho para los retablos colaterales del Altar mayor, creemos que se trata de los dos lienzos de Santa Inés y Santa Catalina realizados por este autor actualmente en el Museo del Prado, donde llegaron procedentes del Museo de la Trinidad.

El inventario realizado en el convento en 1836 por los comisionados de la Academia, describe así estos dos cuadros en la iglesia:

Santa Catalina, de cuerpo entero, con la cabeza del tirano debajo del pie izguierdo. Santa Ynés, de cuerpo entero, con el cordero sobre un libro.

$=0$

Cada uno de ellos mide 7 pies y medio de alto por 4 y medio de ancho, es decir, 2,08 x 1,25 mts., aprox. ${ }^{18}$ y ambos llevan al margen en el inventario la calificación de «medianos», Por sus dimensiones y características, estos lienzos se corresponden sin duda con los números 4.916 (Santa Catalina) y 4.917 (Santa Inés) del inventario actualizado del Museo del Prado. Ambos responden a la descripción ofrecida en el inventario de 1836 y miden 2,13 x 1,26 mts. y 2,12 x 1,25 mts. respectivamente, medidas que casi coinciden totalmente con las expresadas en pies. El segundo de ellos, está además firmado por Vicente Carducho en 1637, fecha muy próxima a la firma de su último contrato con el convento que como vimos data de noviembre de 1634. Desde 1880, ambos lienzos están depositados en la Diputación Provincial de Albacete.

El motivo de que estas dos santas ocupen un lugar destacado en la iglesia del convento, se debe a su condición de patronas de la Orden trinitaria, junto con la Virgen del Remedio. El patronazgo de santa Inés se apoya en que el día 28 de enero de 1193, festividad de santa Inés secundo ${ }^{19}$, recibió San Juan de Mata en el transcurso de su primera misa la revelación divina que lo llevaría a fundar esta orden religiosa.

En cuanto a las razones del patronazgo de santa Catalina de Alejandría no están tan claras, aunque según algunas antiguas Crónicas, el día de su festividad se ordenó San Juan de

16 Mary Crawford Volk, op. cit., p. 283 y 284. La carta de pago se reproduce en la p. 342.

17 Archivo de la Real Academia de Bellas Artes de San Fernando, leg. 35-7/1: Ynventario formado por la Comisión de Nobles Artes pedida a la Real Academia de San Fernando, compuesta de los señores directores de la misma, D. José de Madrazo, D. Juan Antonio Ribera y D. Francisco Elías, en el convento de trinitarios descalzos de esta Corte.

181 pie castellano: $28 \mathrm{cmts}$. aprox.

19 La fiesta litúrgica de Santa Inés se celebraba el día 21 de enero. Anteriormente, la Iglesia celebraba también la octava de esta festividad, día 28, que se conocía con el nombre de Santa Inés secundo. 
Mata de misa, como constaba en un antiguo breviario ${ }^{20}$, por lo que también esta festividad se celebraba de un modo especial.

Por tanto las representaciones de ambas santas en los conventos trinitarios eran muy habituales y frecuentemente en lugares destacados de la iglesia: en el retablo mayor se hallaban en los ex-conventos trinitarios de Ronda (Málaga) y Torrejón de Velasco (Madrid); en la bóveda de la capilla mayor del ex-convento de trinitarios calzados de Cuéllar (Segovia) y otros muchos ejemplos.

En cuanto al tipo de representaciones, solían aparecer como en este caso, en figura aislada, en ocasiones ataviadas con el hábito trinitario (el ya citado ejemplo del convento de Cuéllar o los dos lienzos que procedentes del ex-convento de trinitarios descalzos de Córdoba se hallan hoy en el Museo de Bellas Artes de esta ciudad) y también eran frecuentes las representaciones con escenas de su martirio como las realizadas por Marco Benefial para el antiguo convento de trinitarios calzados de Roma.

Por tanto, el programa iconográfico desarrollado en la cabecera de la iglesia de este convento de trinitarios descalzos de Madrid, quedaría configurado por la Santísima Trinidad, flanqueada por los santos fundadores de la Orden y dos escenas indeterminadas de la vida de éstos, y las dos patronas de la misma, Santa Inés y Santa Catalina.

b) Serie sobre la vida de San Juan de Mata

El contrato entre el convento y el pintor se realizó en 28 de noviembre de 1634. En el documento se especifica que los lienzos ... an de ser de diez pies de alto digo an de ser de ocho y medio poco más o menos y de ancho ocho y quarto asimismo poco más o menos, según las medidas que dice el Padre fr. Francisco de San Joseph.... por precio y quantía de mil y dozientos reales en vellón cada uno con calidad de que su tasación llegue a mil y quinientos reales... ${ }^{21}$.

De esta serie que constaba como dijimos de 12 lienzos, se conservan en la actualidad todos menos uno, destruido en 1936 en la parroquia del Purísimo Corazón de María, de Madrid, donde había sido depositado por el Museo del Prado en 1884. Representaba a San Juan de Mata curando a un endemoniado y su número en el inventario manuscrito del Museo de la Trinidad era el 614.

De los cuadros que pasaron al Museo de la Trinidad, diez se incorporaron posteriormente al Prado; otros dos fueron devueltos en 1861 al Infante D. Sebastián Gabriel de Borbón y Braganza cuya colección había sido requisada en $1835^{22}$. Uno de ellos, que representa la Ordenación y primera misa de San Juan de Mata, se halla actualmente en la Colección Estrany de Palma de Mallorca y el otro, Muerte de San Juan de Mata, pertenece a Patrimonio Nacional.

Al consultar el inventario realizado en el convento por los miembros de la Academia de San Fernando que ya citamos anteriormente, llama la atención la ausencia de estos lienzos.

La razón es que se hallaban escondidos junto con otros cuadros del.convento, entre los que se hallaban algunos de los que conformaban la serie sobre la vida de la Virgen que Ponz citó en el claustro, en casa del duque de Medinaceli. Así, el 14 de abril de 1837, el secretario

${ }^{20}$ Fr. Francisco de Vega y Torayo (O.SS.T.), Ave María. Chrónica de la provincia de Castilla, León y Navarra de la Orden de la Santísima Trinidad, redención de cautivos, Madrid, José Rodríguez de Escobar, 1720, Vol. I, p. 29.

21 Mary Crawford Volk, op. cit., p. 242.

22 Sobre los cuadros de esta serie en la colección del Infante, véase Mercedes Agueda, «La colección de pinturas del Infante Don Sebastián Gabriel de Borbón» en Boletín del Museo del Prado, 1982, pp. 102-117. 
de la Academia D. Marcial Antonio López, comunica por carta al duque que los encargados de recoger los efectos artísticos de la iglesia de este convento habían visto junto al camarín una estancia en la que se hallaban junto con objetos que no parecían pertenecer a religiosos, ... veinte y seis quadros que se echan de menos en el Inventario.

Hechas las pertinentes averiguaciones, se descubrió que aquellas estancias pertenecían a Medinaceli, a espaldas de cuyo palacio se levantaba el convento de trinitarios ${ }^{23}$. El 18 de abril del citado año, el duque contestaba al secretario de la Academia que dichas habitaciones formaban la armería de su casa y que los cuadros se los habían llevado allí los religiosos en 17 de julio de 1834 ante el peligro de saqueo o incendio que se cernía sobre el edificio. Los días 9 y 13 de junio de 1838, pasaron a recogerlos a casa del duque de Medinaceli Arnedo y V. Carderera para llevarlos al Museo de la Trinidad. No se llevaron todos los cuadros, tan sólo los que consideraron mejores, quedando los que se juzgaron de ningún mérito a cargo de Medinaceli que los entregaría en caso de ser reclamados por la Junta directiva del Museo ${ }^{24}$.

Conocemos la lista de los cuadros que fueron llevados al Museo de la Trinidad (20 en total) pero no los que se quedaron en casa del duque. De estos 20 cuadros, sólo diez pertenecían a la serie de San Juan de Mata, hallándose los dos restantes ya en el Museo de la Trinidad con la requisada colección del Infante D. Sebastián, quien pudo adquirirlos en 1834, coincidiendo con el traslado de la serie a casa de Medinaceli. Como hemos visto, los religiosos habían guardado allí las obras que probablemente consideraron más valiosas: la serie del claustro y la de la vida de San Juan de Mata, ambas trasladadas a la Trinidad. Es probable que también se escondieran aquí los cuadros de Carducho para el retablo mayor, ya desmontado, y que no fueron al Museo de la Trinidad.

Constituyen estos lienzos de la vida de San Juan de Mata un conjunto perfectamente conocido y citado por los antiguos tratadistas; además de Ponz, también los mencionan Palomino ${ }^{25}$ y Ceán ${ }^{26}$.

Una vez incorporados al Museo de la Trinidad, el Catálogo de Cruzada Villaamil realizado en 1865 cita sólo siete de los diez lienzos que quedaban (los otros dos ya habían sido devueltos al Infante), aunque sin considerarlos como parte de un conjunto y sin identificar en algunos casos los temas representados en ellos, catalogados como «asunto místico», incluyendo dos de ellos entre los anónimos ${ }^{27}$.

Lo mismo sucede en el artículo de Gaya Nuño sobre el Museo de la Trinidad: sólo se citan algunos cuadros de manera aislada, y señalando la procedencia de un convento trinitario únicamente de tres de los lienzos ${ }^{28}$.

Son Diego Angulo y Alfonso E. Pérez Sánchez quienes en 1969 en su obra sobre pintura madrileña ${ }^{29}$ identifican por primera vez estos cuadros con los de la serie del convento de trinitarios descalzos de Madrid, creyéndolos realizados hacia 1632 que es cuando el pintor, como vimos, se compromete a pintar el retablo mayor de la iglesia, lo que explicaría las similitudes estilísticas entre los cuadros de esta serie y los realizados por Vicente Carducho para la Cartuja de

23 A.R.A.B.A.S.F., leg. 35-23/1.

24 Id., leg. 35-20/1.

25 Antonio Palomino, Vidas. Madrid, Aguilar, 1988, p. 148.

26 J.A. Cean Bermúdez, Diccionario, 1965, Vol. I, p. 255.

${ }_{27}$ Gregorio Cruzada Villaamil, Catálogo provisional, historial y razonado del Museo Nacional de Pinturas, Madrid, 1865, números 112, 114, 482, 608, 633 y 638.

28 J. A. Gaya Nuño, «El Museo Nacional de la Trinidad. Historia y catálogo de una pinacoteca desaparecida» en Boletín de la Sociedad Española de Excursiones, 1947, pp. 20-77.

29 Diego Angulo Iñíguez y Alfonso E. Pérez Sánchez, Historia de la pintura española. Pintura madrileña del primer tercio del siglo XVII. Madrid, C.S.I.C., 1969, pp. 146-151 números 333-343. 
El Paular, de fecha muy próxima. Ambos autores recogen toda la bibliografía existente hasta la fecha acerca de esta serie e identifican correctamente los temas desarrollados en los lienzos (sólo catalogan once, pues todavía se ignoraba la existencia de otro cuadro perteneciente a esta serie) tomando como base el texto de una Crónica contemporánea a la realización de la misma. En dicha Crónica se inserta un grabado con escenas de la vida de San Juan de Mata firmado por Jean de Courbes y Fr. Cristóbal Rolo (probablemente el religioso encargado de diseñar el programa iconográfico que después representa el grabador) que, como ya señalaron Angulo y Pérez Sánchez, presenta los temas de modo análogo al de Carducho ${ }^{30}$.

Con posterioridad a la obra de Angulo y Pérez Sánchez destaca el trabajo ya mencionado de Mary Crawford, que sigue citando sólo once lienzos.

Pérez Sánchez dio a conocer en 1980 el cuadro que faltaba para completar la serie: se trata del Encuentro entre San Juan de Mata y San Félix de Valois, depositado por el Museo del Prado en el Consejo de Estado (Madrid) desde $1883^{31}$.

Además de estas aportaciones bibliográficas recientes, existe otro punto que puede considerarse interesante para mejorar el conocimiento acerca de este conjunto, y que no ha sido citado todavía por ninguno de los autores que se han ocupado del mismo. Se trata de la influencia de una serie de grabados realizados por Theodore van Thulden para el convento de St. Mathurin de París, de trinitarios calzados, en 1633, que recogen las escenas de la vida de los santos fundadores pintadas por el mismo autor para decorar la iglesia del convento.

Ya Jeaninne Baticle habló de las similitudes entre la composición empleada por Carreño de Miranda en su gran cuadro para el altar mayor de trinitarios descalzos de Pamplona (Fundación de la orden trinitaria, hoy en el Louvre) y la escena que recoge este mismo tema en la serie de grabados de Thulden ${ }^{32}$

En otras series de la Vida de San Juan de Mata realizadas en el siglo XVIII, se siguen percibiendo influencias de estos grabados, que debieron tener una gran difusión ${ }^{33}$.

Los cuadros realizados para St. Mathurin por Theodore van Thulden (1606-1676) pintor y grabador, discípulo de Rubens, desaparecieron con el resto del edificio durante la Revolución francesa. Afortunadamente, estas composiciones fueron recogidas en una serie de 24 grabados publicados en París en 1633, de los que existe un ejemplar completo en la Biblioteca Nacional de Madrid ${ }^{34}$.

Los grabados vienen precedidos por un prólogo en latín en el que se explica que el entonces General de los trinitarios, Fr. Louis Petit, encargó al pintor decorar la iglesia conventual con escenas alusivas a la «revelación angélica» de esta orden y a los primeros padres

${ }^{30}$ Se trata de la obra de Fr. Pedro López de Altuna (O.SS.T.), Crónica general de la Orden de la Santísima Trinidad, redención de cautivos, Segovia, Pedro Díez de Escalante, 1637.

31 Alfonso Emilio Pérez Sánchez, «Un lienzo de Vicente Carducho, identificado» en Boletín del Museo del Prado, 1980, pp. 159-162.

32. Jeaninne Baticle, «La Fundación de la Orden trinitaria de Carreño de Miranda» en Goya, 1964, pp. 141-153.

33 El Museo Toledano de Santa Cruz conserva un lienzo de la primera mitad del siglo XVII, de autor anónimo español, que representa la Muerte de un fraile trinitario. El cuadro es copia de un original perdido de Van Thulden y es exacto a la estampa correspondiente a esta escena incluida en la serie de que estamos hablando.

${ }^{34}$ Revelatio Ordinis SS. Trinitatis Redemptionis captivorum sub Innocentio Tertio anno 1198, Biblioteca Nacional, Estampas, E.R.- 1.541. Sobre estos grabados, véase Hollstein's dutch and flemish etchings, engravins and woodcuts. ca. 1450-1700, Amsterdam, (s.a. 1954?), vol. XXX, pp. 108-111, números 12-35. Los ejemplares de esta serie existentes en París y Bruselas llevan inserto otro grabado que representa a St. Mathurin expulsando a los demonios. También citan este ciclo de San Juan de Mata J. Vallery-Redot, «Note sur les estampes gravées par Mellon y Van Thulden pour les Ordres redempteurs de N.D. de la Merci et des Trinitaires» en Bulletin philologique et historique (Jusq'a 1715) du Comité des travaux historiques et scientifiques, 1953-1954, pp. 417-421, y E. Escallier, Le liberateur des esclaves ou le redempeteur des captifs S. Jean de Matha, Raphele-les-Arles (Bouches du Rhône), 1960,'pp. 149-160. 
adoradores de la Trinidad y después le encargó grabar estas escenas en planchas de cobre. Algunas afirmaciones de las contenidas en este texto introductorio, son significativas respecto a la finalidad que se quería dar a los grabados, que se consideraban una obra útil para contribuir al mejor conocimiento y difusión de los temas en ellos contenidos.

Las similitudes entre algunos de los cuadros que conforman la serie de Carducho (contratada un año después de la aparición de los grabados) y las estampas de Thulden, son evidentes por lo que parece lógico pensar que Carducho las conocía y utilizó, lo que pudo suceder porque alguno de estos libros conteniendo los grabados llegase a sus manos ${ }^{35}$ o más probablemente, a través del Prior o algún religioso del convento de Madrid.

Los cuadros en los que percibimos más claramente influencia de los grabados de Theodore van Thulden son los siguientes:

\section{Milagroso anuncio del nacimiento de San Juan de Mata ${ }^{36}$}

Entre 1882 y 1980 estuvo depositado en el Museo de Valladolid, y desde 1986 se encuentra en el Museo municipal de Játiva (Valencia).

Representa la aparición de la Virgen a la madre del santo para anunciarle la santidad de su futuro hijo. En el Catálogo de la Trinidad realizado por Cruzada Villaamil se cita como «Anuncio del nacimiento de una santa» y Gaya Nuño lo llama «Asunto místico».

Las semejanzas con el grabado se han trasladado al segundo plano en el cuadro. En éste, la hermosa figura de la Virgen aparece entre nubes y angelitos dirigiéndose a la madre del santo, que está arrodillada ante ella. Este grupo de primer plano ha sido sustituido en el grabado por tres mujeres que bañan al recién nacido en una pila y otra que seca unos paños en la chimenea. En el cuadro, Carducho ha colocado también en segundo término a läs mujeres bañando al niño y la mujer secando los paños, en esta ocasión ante un brasero. En este lado derecho el fondo se dispone igual en ambos casos: una cama con dosel donde se halla la madre del santo a quien atiende una mujer sentada a su lado, y una puerta junto a ella (Figs. 2 y 3 ).

\section{San Juan de Mata se despide de sus padres ${ }^{37}$}

El lienzo estuvo depositado entre 1883-1981 en el Museo de Villanueva y Geltrú (Barcelona) y actualmente se halla en el Museo del Prado. Representa al santo en el momento de abandonar la casa paterna para marcharse a estudiar a la universidad de París.

Gaya Nuño lo llama «personaje arrodillado a los pies de un santo» y Angulo y Pérez Sánchez subrayaron las semejanzas entre el personaje arrodillado que aquí representa a San Juan de Mata y el San Bruno de la escena «Humildad y desprecio de las cosas terrenales en

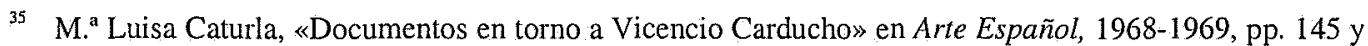
ss., publica el inventario de bienes del pintor realizado en 1638. Entre los libros, sólo se citan dos que pudieran ponerse en relación con esta serie: un Compendio de Trinitarios y una Vida de San Juan de Mata, ambos tasados en tres reales. Por la fecha en que se hace este inventario, el primer libro se trata seguramente de la obra de Gil González Dávila, Compendio histórico de las vidas de los gloriosos San Juan de Mata y San Félix de Valois... Madrid, 1630. En cuanto a la Vida de San Juan de Mata, ignoramos de qué libro puede tratarse. La primera Crónica de la Orden en España de que tenemos noticia es la ya citada obra del P. López de Altuna, que como dijimos se publica en 1637. Aunque es una Crónica general de la Orden, la primera parte contiene la vida de San Juan de Mata y San Félix de Valois.

${ }^{36}$ Museo de la Trinidad n. ${ }^{\circ}$ 633; Inv. actualizado Museo del Prado n. ${ }^{\circ}$ 5.450; Cruzada, op. cit., p. 22, n. ${ }^{\circ}$ 633; Gaya Nuño, op. cit., p. 36; Angulo y Pérez Sánchez, p. 147, n. ${ }^{\circ} 333$; Crawford, op. cit., p. 244, n. ${ }^{\circ} 83$.

37 Museo de la Trinidad n. ${ }^{\circ} 422$; inv, actualizado Museo del Prado n. ${ }^{\circ}$ 5.089; Gaya Nuño, op. cit., p. 36; Angulo y Pérez Sánchez, op. cit., p. 147, n..$^{\circ} 334$; Crawford, op. cit., p. 245, n. ${ }^{\circ} 84$. 
la orden cartujana» de la serie de El Paular, lo que revela la utilización del mismo dibujo en ambos casos.

Nuevamente, algunos detalles empleados por el pintor así como la composición general de la escena remiten al grabado correspondiente de la serie de Thulden: así, la figura de la mujer llorando que se acerca un pañuelo al rostro y la misma figura del padre del santo, un hombre alto y barbado, que en el grabado estrecha la mano de su hijo, montado ya en el caballo, y en el cuadro realiza ante áquel arrodillado, un signo de bendición. En ambos casos el fondo de la escena está ocupado en una mitad con arquitectura y en otra con paisaje, introduciéndose elementos anecdóticos como un perrillo en primer plano y una escultura, que en el caso de Thulden es también una fuente y que en el cuadro aparece inserta en una hornacina (Figs. 4 y 5 ).

\section{Encuentro de San Juan de Mata y San Félix de Valois ${ }^{38}$}

Corresponde al n. ${ }^{\circ} 543$ del inventario de la Trinidad y n. ${ }^{0} 3.345$ del inventario actualizado del Museo del Prado. Desde 1883, se halla depositado en el Consejo de Estado.

La escena recoge el instante en que San Félix de Valois, que se había retirado a vivir como ermitaño, recibe a San Juan de Mata que por inspiración divina había acudido a su encuentro, decidiendo posteriormente los dos la fundación de esta orden religiosa.

Gaya Nuño lo cita en su estudio sobre los cuadros de la Trinidad, pero hasta hace pocos años, el cuadro no había sido identificado como parte de esta serie, dándolo a conocer el Dr. Pérez Sánchez en 1980 en el artículo que citamos con anterioridad. Señala el autor las semejanzas entre este lienzo y el «San Antonio Abad y San Pablo primer ermitaño» de Velázquez que se halla en el Museo del Prado aunque es difícil determinar a quién corresponde la prioridad en el uso de este esquema compositivo, así como la existencia de un dibujo preparatorio para la figura de San Félix de Valois arrodillado, que se halla en la Biblioteca Nacional de Madrid. La escena será retomada en 1665 por Carreño en la realización de su gran cuadro para el altar mayor de trinitarios descalzos de Pamplona del que ya hablamos, aunque reduciéndola a escenita en segundo término, donde hallamos a los dos santos sentados en idéntica disposición que en este cuadro de Carducho.

El pintor ha situado en el centro el momento en que se encuentran los dos santos: San Juan de Mata aparece acompañado por el ángel que lo guió hasta San Félix de Valois, que se arrodilla ante él. Al fondo a la derecha, entre el paisaje, los dos santos parecen escuchar al ángel que los advierte de la necesidad de ir a Roma a contar al Pontífice su milagrosa visión (la aparición de un ciervo con una cruz entre las astas que aquí se ha colocado junto a la fuente) ${ }^{39}$.

${ }^{38}$ Museo de la Trinidad n. ${ }^{\circ} 543$; Inv. actualizado Museo del Prado n. ${ }^{\circ} 3.345$; Gaya Nuño, op. cit., p. 73, n. ${ }^{\circ}$ 343; Pérez Sánchez, op. cit., pp. 159-162.

39 La fundación de la Orden Trinitaria tuvo su origen en la milagrosa visión de San Juan de Mata. Celebrando el santo su primera misa, suplicaba fervorosamente al Señor le indicase la Orden en que debía ingresar. Al llegar el momento de la consagración, Cristo se le apareció con dos cautivos, llevando en el pecho cada uno de éstos una cruz rojiazul, revelación divina que le llevaba a fundar una orden dedicada a la redención de los cautivos cristianos. Por su parte, retirado San Félix de Valois en el yermo, recibió durante varios días la visión de un ciervo que tenía entre las astas una cruz asimismo rojiazul y cuando se reunió con él San Juan de Mata, ambos volvieron a tener esta misma visión, que se representa en el cuadro de Carducho, lo que les decidió a marchar a Roma para informar al Papa acerca de sus proyectos de fundación. 

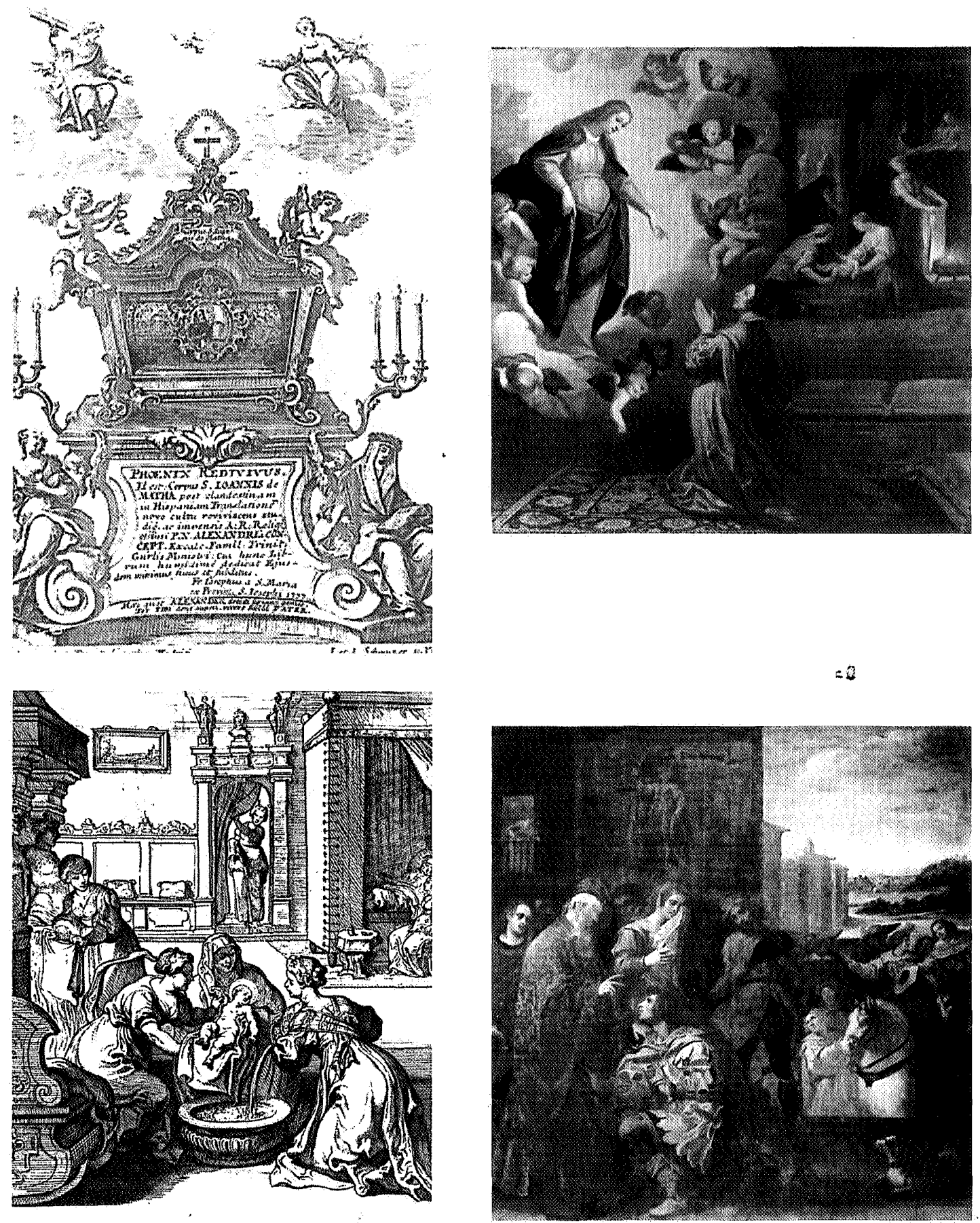

Wafectur feliciter B. To.sNNEs de Aratta in Falcone oppid af Mlper Prouncia in Gallia. Pater Eyfremius de Matta,

Figuras 1-4. Urna con las reliquias de San Juan de Mata. Grabado realizado por I. et A. Schmuzer a partir del cuadro del mismo tema por Fr. Bartolomé de San Antonio (Viena, 1733). Vicente Carducho: Milagroso anuncio del nacimiento de San Juan de Mata. Játiva (Valencia) Museo Municipal (Depósito Museo del Prado). Theodore van Thulden: Nacimiento de San Juan de Mata. Madrid, Biblioteca Nacional. Vicente Carducho: San Juan de Mata se despide de sus padres. Madrid, Museo del Prado. 

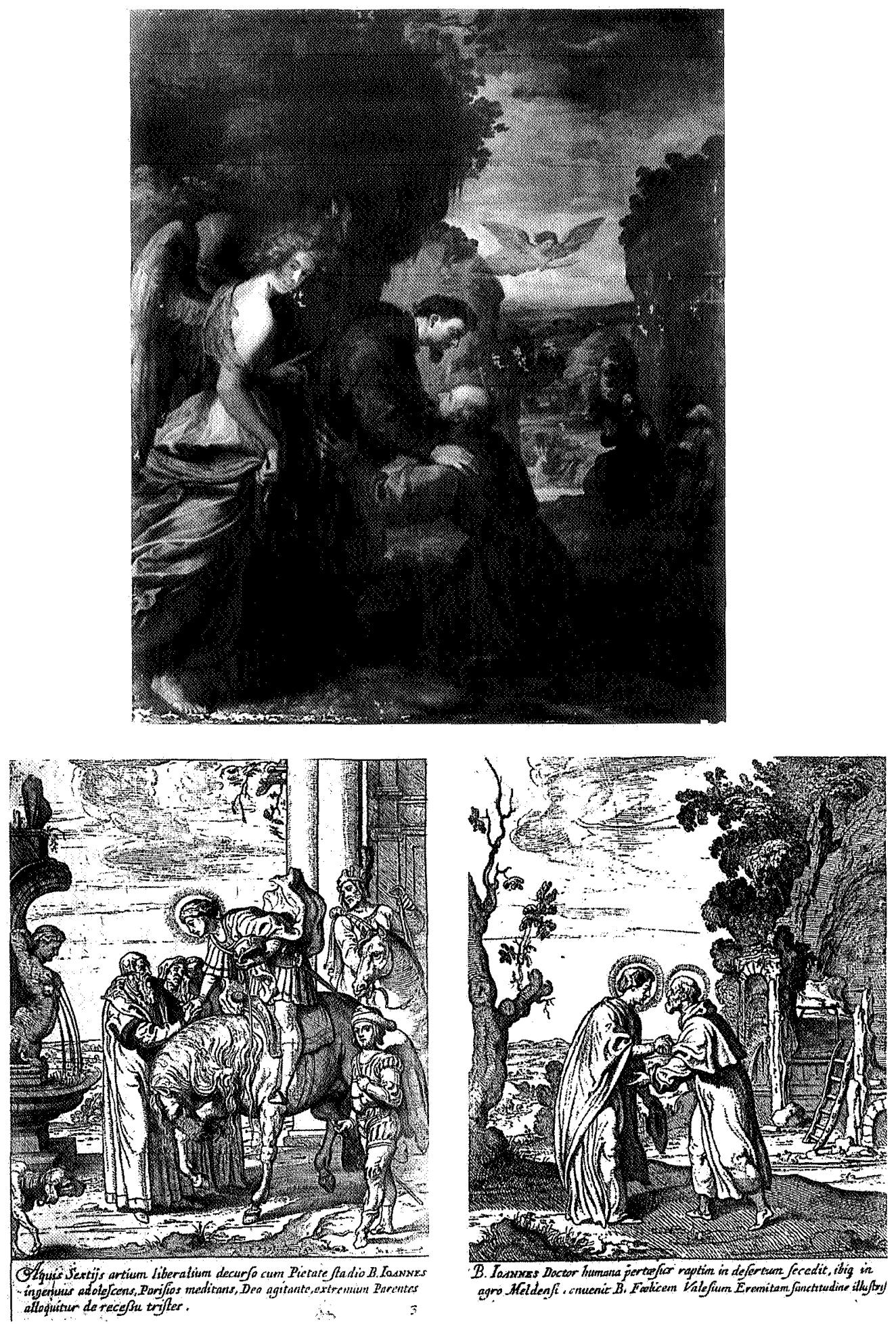

Figuras 5-7. Theodore van Thulden: San Juan de Mata se despide de sus padres. Madrid, Biblioteca Nacional. Vicente Carducho: Encuentro de San Juan de Mata y San Félix de Valois. Madrid, Consejo de Estado (Depósito Museo del Prado). Theodore van Thulden: Encuentro de San Juan de Mata y San Félix de Valois. Madrid, Biblioteca Nacional. 
En el grabado correspondiente de la serie de Van Thulden, la escena se desarrolla en un marco muy similar. Los dos religiosos (San Juan de Mata con un sombrero en la mano) estrechan sus manos ante un fondo de paisaje que al contrario de lo que sucedía en el cuadro de Carducho, se abre por el lado izquierdo. En el lado derecho, llenándolo casi por completo, se disponen árboles, rocas y la fuente junto a la que tendría lugar la visión. Estos mismos elementos de paisaje, junto con el ángel, llenan el cuadro en el hueco de la izquierda (Figs. 6 y 7 ).

\section{Visión de Inocencio III y confirmación de la orden trinitaria ${ }^{40}$}

Desde 1882 está depositado por el Museo del Prado en el Museo de Valladolid.

Representa la milagrosa visión del Papa Inocencio III a quien se le apareció un ángel con dos cautivos durante la celebración de una misa, en la misma forma que Cristo se había mostrado a San Juan de Mata. Después de esta visión el Papa se decidió a aprobar el nacimiento de la Orden ${ }^{41}$.

Gaya Nuño cita el lienzo como «San Juan de Mata profesando en la Orden».

Es quizá en esta escena donde la similitud entre el cuadro de Carducho y el grabado de Thulden se aprecia con mayor claridad. La composición se concibe en ambos casos de manera casi idéntica: el Papa se sitúa de espaldas al espectador en el momento de elevar la Sagrada Forma y contemplar en milagrosa visión al Angel de la Redención. A su lado, dos personajes arrodillados sostienen el manto. En ambos extremos se disponen miembros de la corte papal. El espacio central, vacío en el grabado, lo ocupan en el cuadro guardias pontificios. En primer plano, se distinguen en el cuadro y en el grabado dos figuras arrodilladas que corresponden a San Juan de Mata y San Félix de Valois, asistentes a la milagrosąamisa celebrada por el Papa: Incluso el fondo arquitectónico colocado detrás del altar es el mismo en ambos casos, aunque en el cuadro se ha incluido, a nuestra derecha, la escena en que Inocencio III una vez aprobada la Orden entrega a los religiosos el nuevo hábito, que también guarda una gran semejanza con el grabado correspondiente en la serie, donde se ha recogido de forma independiente (Figs. 8 y 9).

\section{San Juan de Mata entregando las cartas del Papa al rey de Marruecos en la primera redención ${ }^{42}$}

Depositado en el Colegio de San Ildefonso (Madrid) desde 1885 hasta 1988, actualmente se halla en el Museo del Prado.

La escena muestra al santo en el momento de entregar las cartas del Papa al rey de Marruecos («miramamolín» o «miramamolino» lo llaman los antiguos cronistas) que se halla acompañado por varios miembros de su séquito, sentado bajo un dosel. En segundo término, a la izquierda, San Juan de Mata negocia con los musulmanes el pago del rescate, mientras señala al cautivo que está arrodillado a sus pies. Al fondo se distingue el barco con los estandartes de la Orden.

En el grabado, la composición es la misma, si bien la escena muestra al santo en el momento de hacer entrega del dinero mientras detrás aguardan los infelices cautivos y en la parte izquierda se distingue también el barco de los redentores (Figs. 10 y 11).

${ }^{40}$ Museo de la Trinidad n. ${ }^{\circ} 608$; Inv. actualizado Museo del Prado n. ${ }^{\circ} 5.454$; Cruzada, op. cit., p. 23 n. ${ }^{\circ} 608$; Gaya Nuño, op. cit., p. 36; Angulo y Pérez Sánchez, op. cit., p. 419 n. ${ }^{\circ} 38$; Crawford, op. cit., p. 249 n. ${ }^{\circ} 88$.

41 Por medio de la Bula Operante divine dispositionis, expedida por Inocencio III a comienzos de diciembre de 1198

${ }^{42}$ Museo de la Trinidad n..$^{\circ}$ 638; Inventario actualizado Museo del Prado n. ${ }^{\circ} 3.346$; Cruzada, op. cit., p. 23 n. ${ }^{\circ}$ 638; Gaya Nuño, op. cit., p. 23; Angulo y Pérez Sánchez, op. cit., p. 149 n. ${ }^{\circ} 339$; Crawford, op. cit., p. 251 n. ${ }^{\circ} 290$. 

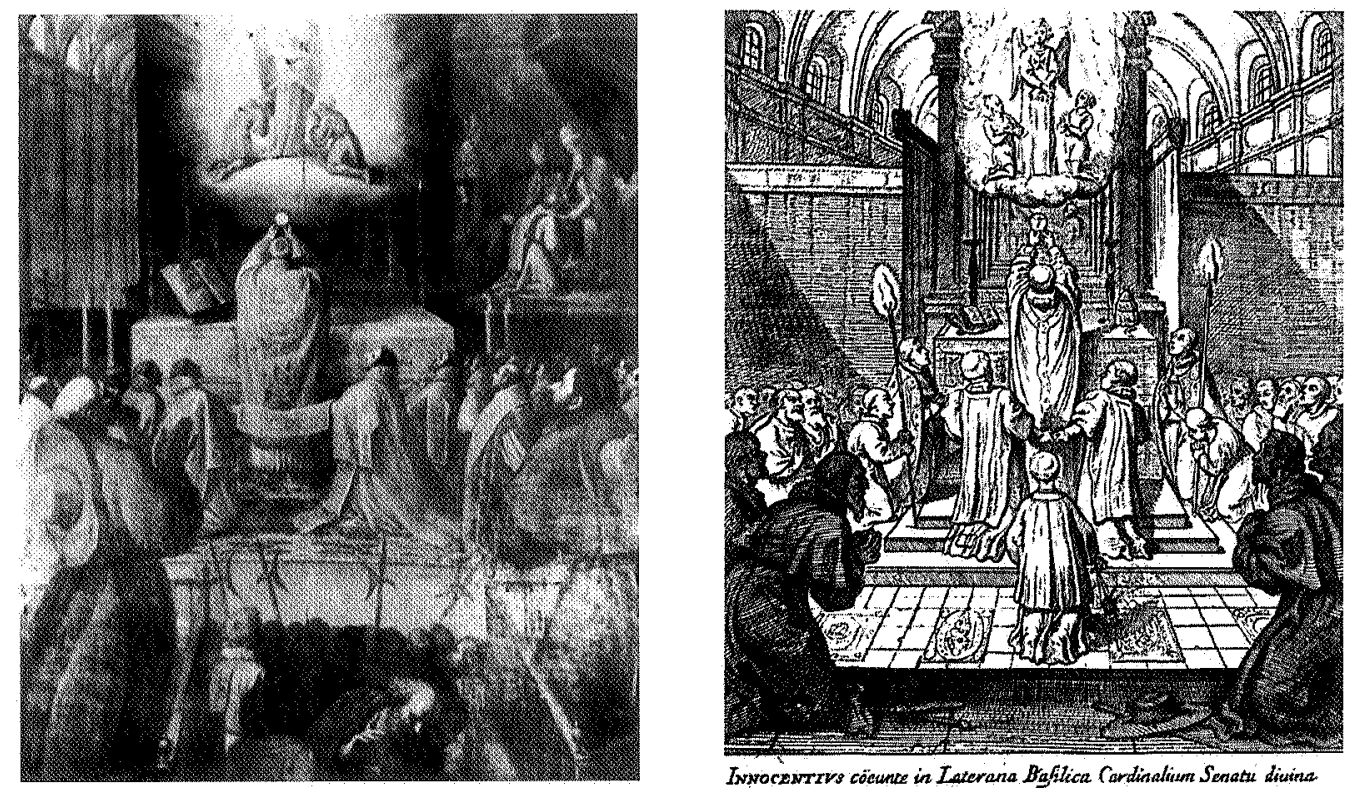

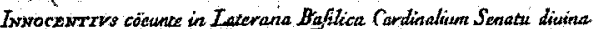
celebrext, apparee Angelus Cruce fignatur cun Captiuis, illos commutont fimilinf felto. D. Agnetis $2:$ an: $y \geqslant \theta$.
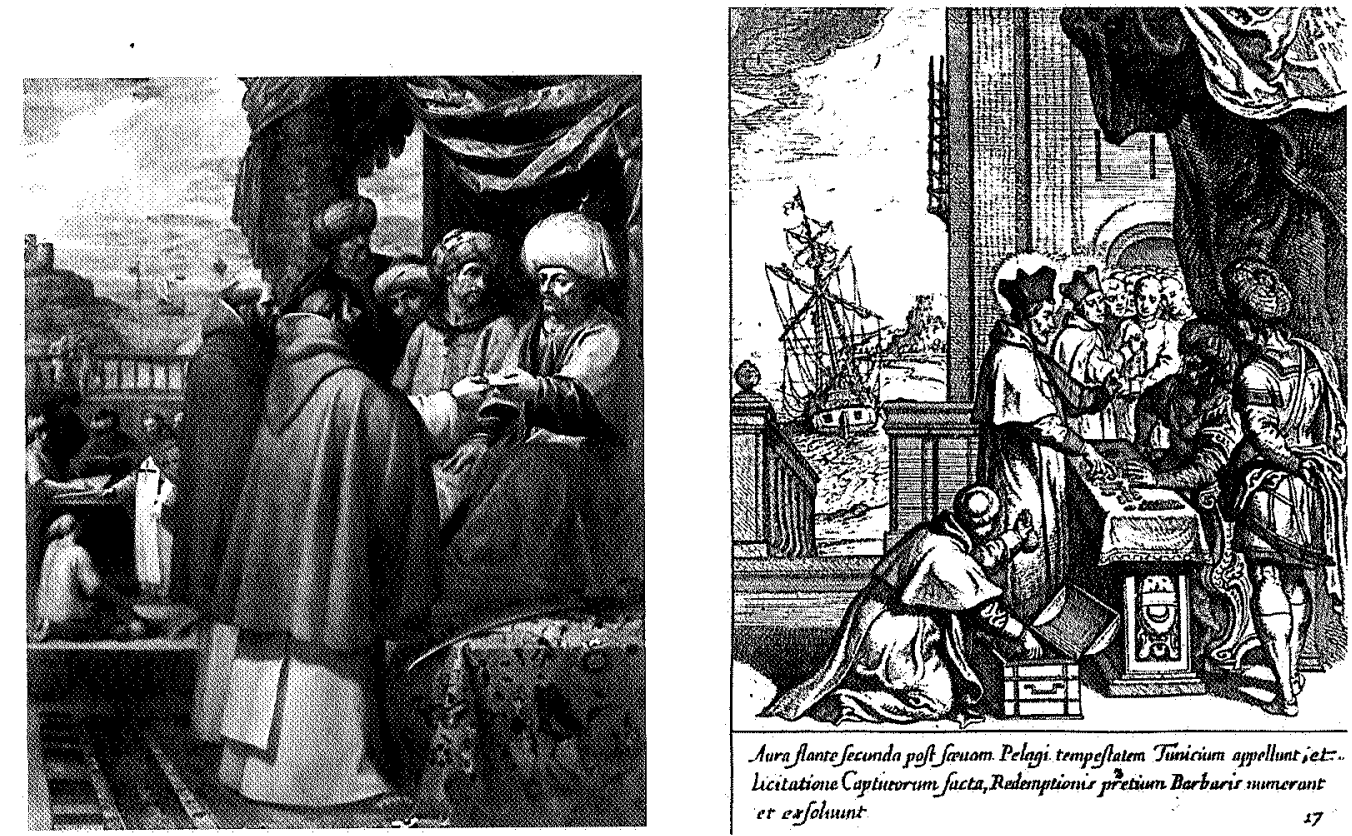

Figuras 8-11. Vicente Carducho: Visión de Inocencio III y confirmación de la Orden Trinitaria. Valladolid, Museo (Depósito Museo del Prado). Theodore van Thulden: Visión de Inocencio III y confirmación de la Orden Trinitaria. Madrid, Biblioteca Nacional. Vicente Carducho: San Juan de Mata entrega las cartas del papa al rey de Marruecos en la primera redención. Madrid, Museo del Prado. Theodore van Thulden: San Juan de Mata entrega las cartas del Papa al rey de Marruecos en la primera redención. Madrid, Biblioteca Nacional. 

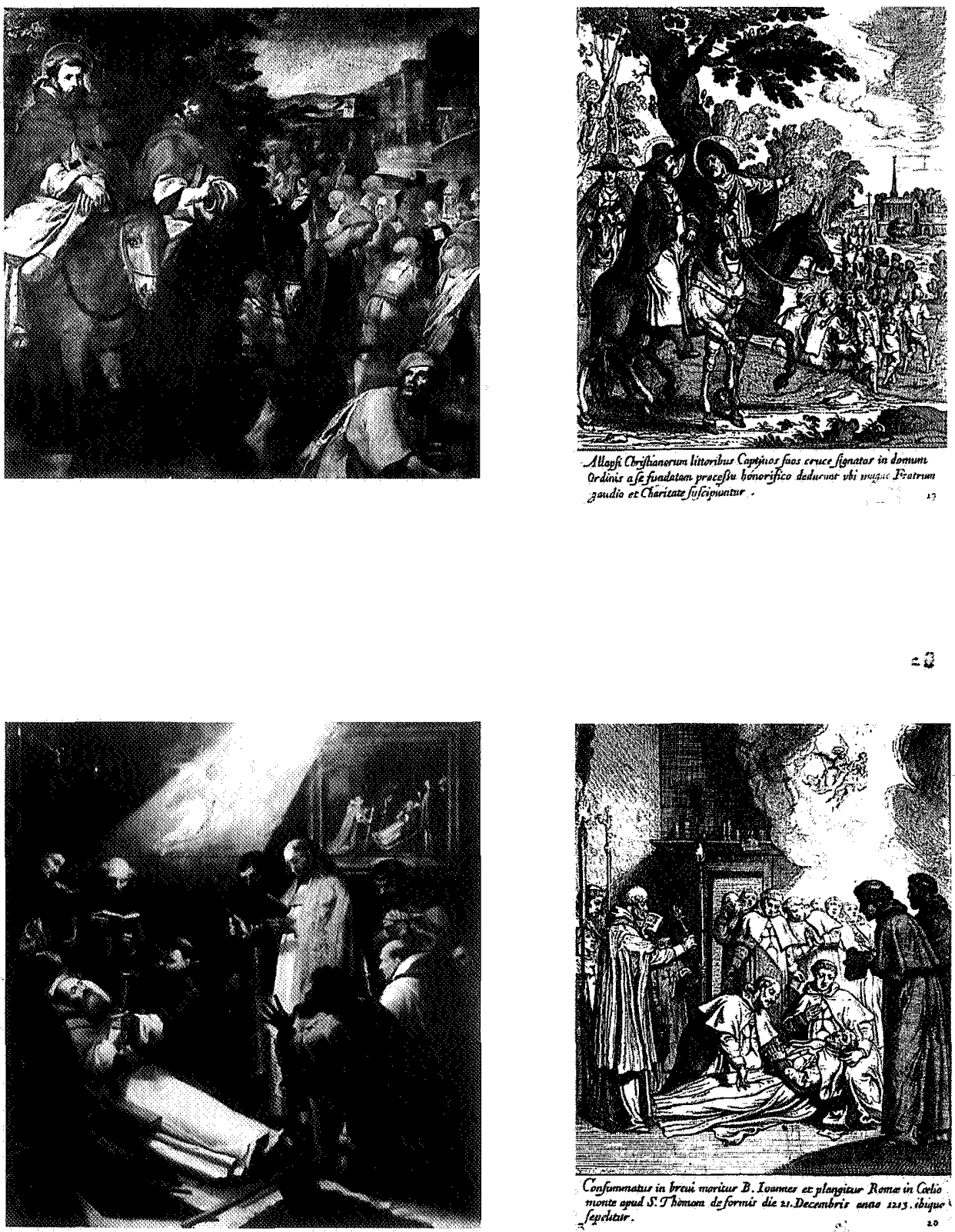

Figuras 12-15. Vicente Carducho: San Juan de Mata regresa con los cautivos liberados. Villanueva y Geltrú (Barcelona), Museo Balaguer (Depósito Museo del Prado). Theodore van Thulden: San Juan de Mata regresa con los cautivos liberados. Madrid, Biblioteca Nacional. Vicente Carducho: Tránsito de San Juan de Mata. Patrimonio Nacional, Palacio Real de Aranjuez (Madrid). Theodore van Thulden: Tránsito de San Juan de Mata. Madrid, Biblioteca Nacional. 


\section{San Juan de Mata regresa con los cautivos liberados ${ }^{43}$}

El cuadro se halla depositado por el Museo del Prado en el Museo Balaguer de Villanueva y Geltrú (Barcelona).

En el Catálogo de Cruzada figura como anónimo, y Gaya Nuño lo cita dos veces: la primera como no catalogado por Cruzada y la segunda diciendo que en dicho Catálogo es el número 635 entre los anónimos madrileños.

En esta ocasión son también bastante claras las semejanzas entre cuadro y grabado: los dos redentores (en el grabado son tres) sobre sus monturas, se disponen sobre fondo de árboles avanzando tras la procesión de cautivos liberados, que en ambos casos se sitúan a la derecha ataviados con el escapulario de la Orden y marchando hacia el convento que se distingue al fondo. En el lienzo el convento ha sido sustituido por la entrada de una ciudad, en la que se han congregado numerosas personas para ver llegar la procesión, distinguiéndose un obispo bajo palio (Figs. 12 y 13).

\section{Tránsito de San Juan de Mata ${ }^{44}$}

Junto con el que representaba la Profesión de San Juan de Mata, pertenecía a la colección del Infante D. Sebastián a quien fue devuelto en 1861. Angulo y Pérez Sánchez y más tarde Mary Crawford lo citan en paradero desconocido. Actualmente pertenece a Patrimonio Nacional y se halla en el Palacio de Aranjuez.

El lienzo representa el tránsito y entierro de San Juan de Mata rodeado por algunos de sus religiosos. El santo murió en el convento romano de Santo Tomás in Formis el 17 de diciembre de 1213, según el Martirologio Romano y una larga tradición interna a la Orden ${ }^{45}$.

La composición empleada aquí por Carducho, es muy similar a la que utiliza Thulden en el correspondiente grabado: en ambos casos, San Juan yace en el suelo rodeado por los religiosos, con las manos sobre el pecho, aunque en el cuadro lleva un crucifijo entre las manos. A sus pies, un sacerdote rocía el cadáver con agua bendita (esta última figura es casi idéntica en ambos casos, incluido el cirio y el crucifijo que se halla detrás del difunto en el cuadro y detrás del sacerdote en el grabado). Junto al sacerdote, otra figura sostiene en ambos casos el recipiente para el agua bendita. En la parte superior del grabado, se aprecia el momento en que, envuelto en nubes de gloria, el cuerpo de San Juan de Mata es trasladado al cielo por un ángel como también observamos en el cuadro si bien en este último caso vemos dos ángeles, dentro de un potente haz de luz que, a modo de foco, desciende sobre el santo.

En el lienzo de Carducho se incluye además otra escenita al fondo, que representa el momento de la muerte de San Juan rodeado por los religiosos.

Es curioso observar en esta escena la diferencia entre los personajes del grabado, vinculado a los trinitarios calzados y al convento más importante que la Orden tuvo en Francia, y los representados por Carducho, trabajando aquí para los recién nacidos trinitarios descalzos madrileños quienes, como hemos visto, comenzaron su andadura como comunidad religiosa en medio de grandes estrecheces. Obsérvese la diferencia entre el santo del cuadro, rodeado por sus instrumentos de penitencia, descalzo, con barba un tanto desaliñada y la tosquedad de sus ropas, que contrastan con las figuras más elegantes en el grabado (Figs. 14 y 15).

${ }^{43}$ Museo de la Trinidad n..$^{\circ}$ 635; Inv. actualizado Museo del Prado n..$^{\circ}$ 5.096; Cruzada, op. cit., p. 112 n. ${ }^{\circ} 635$; Gaya Nuño, op. cit., pp. 36 y 66; Angulo y Pérez Sánchez, op. cit., p. 149 n. ${ }^{\circ} 430$; M. Crawford, op. cit., p. 250 n. ${ }^{\circ} 89$.

${ }_{44}$ Museo de la Trinidad n. ${ }^{\circ}$ 294; Angulo y Pérez Sánchez, op. cit., p. 151 n. 343 , Crawford, op. cit., p. 284; M. Agueda, op. cit., n. ${ }^{\circ} 11$.

${ }_{45}$ Juan Pujana, (O.SS.T.), La Orden de la Santísima Trinidad, Salamanca, Secretariado Trinitario, 1993, p. 33. 